
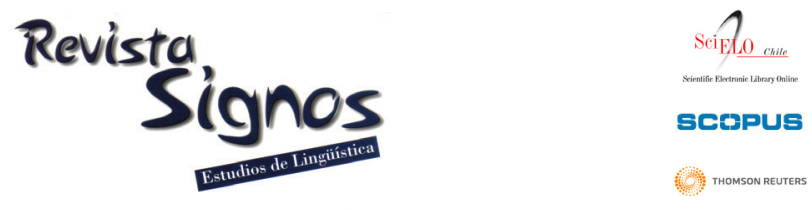

\title{
Ramírez, R. (2010) \\ Didácticas de la lengua y de la argumentación escrita
}

260 pp. Pasto (Colombia): Universidad de Nariño ISBN: 978-958-8609-19-5

\section{Mireya Cisneros}

Universidad Tecnológica de Pereira

Colombia

mireyacealfalcol@yahoo.es

La enseñanza de la lengua en su forma oral y escrita constituye un tema de permanente actualización. De ahí que esta obra -aunque no aborda, de manera directa, la argumentación oral, aspecto fundamental y muy descuidado en los distintos niveles de escolaridad-por un lado, se consolida en un aspecto esencial en la actividad educativa, y, por el otro, en un enfoque renovador a partir de la construcción de un marco conceptual que facilita tanto la enseñanza como el aprendizaje lingüístico concretamente de la argumentación escrita, uno de los aspectos más débiles en los proyectos educativos contemporáneos.

Las distintas posiciones didácticas que puede contener una obra de didáctica de la lengua escrita en la que pueden cimentarse las bases para desarrollos posteriores, en esta obra se abordan de una forma equilibrada, tanto desde el punto de vista intensivo, como extensivo. En cada uno de sus capítulos presenta las distintas posibilidades que ofrece el actual estado del arte, y desfilan autores clásicos y contemporáneos; pero no se trata de solo un compendio sino de la maestría de ponerlos a dialogar entre sí, a reflexionar y a confrontar los distintos planteamientos para guiar las búsquedas en torno a la enseñanza y aprendizaje de la didáctica de la lengua escrita, teniendo en cuenta el desarrollo evolutivo y las necesidades de los estudiosos, en coherencia con 
los constructos teóricos de las ciencias del lenguaje, la comunicación y la educación con una visión interdisciplinaria y transdisciplinaria. Además, a lo largo de toda la obra está el aporte propio del autor quien es una persona con una amplia experiencia como docente y autor de diversos trabajos dedicados al estudio de la didáctica de argumentación (Ramírez, 2003, 2004, 2005a, 2005b, 2008).

El Capítulo I, Marco educativo-profesional de la argumentación, se consolida con aportes de la sociología y la psicología. La educación, en particular la enseñanza del lenguaje, durante el siglo XX han tenido fundamentos transdisciplinarios que caracterizan los diferentes modelos, los cuales se han sucedido hasta alcanzar los fundamentos pragmáticos de los proyectos más actuales y con consenso en la comunidad educativa. Así, en este capítulo, se reflexiona sobre los principios de las ciencias de la educación como recursos que facilitan los estudios de la argumentación en la escuela, con la fundamentación en autores de la escuela nueva, del antiautoritarismo, de las perspectivas marxistas y de los enfoques comunicativos. Igualmente, se toma en cuenta las teorías piagetanas, vigotskianas y el Programa de Inteligencia Práctica en la Escuela, con el objeto de precisar cómo el cerebro del individuo gesta la recreación de la ciencia en la teoría y en la práctica. Finaliza este capítulo asumiendo la noción de transposición didáctica para evidenciar cómo los saberes científicos sobre argumentación se transforman en saberes escolares que pueden ser enseñados.

El Capítulo II, Didáctica de la lengua escrita, se centra en la enseñanza de la lengua, campo de aplicación en el cual suelen presentarse muchos de los problemas, aún sin resolver, que afectan el panorama educativo contemporáneo. Uno de ellos sería escribir sin lecturas, práctica que establece la distancia cultural respecto del libro. Si bien el libro salta del papel al medio digital o virtual, en nada comporta la desaparición de la lectura y, por consiguiente, de la escritura que, como sucedió con la aparición de la imprenta, queda fortalecida porque las nuevas tecnologías también representan nuevas razones para leer y escribir. Así, en este capítulo se analiza la estructura y la proyección de la didáctica de la lengua y lo que puede ser la didáctica de la escritura: objetivos de estudio, procedimientos, actitudes y actividades de aprendizaje. Se plantea un breve análisis de la evolución de la didáctica de la lengua con el propósito de representar el camino que ha recorrido la disciplina hasta lo que se conoce como enfoque comunicativo de la enseñanza de la lengua. Se precisa lo que se entiende por una didáctica de la escritura, de tal suerte que se pueda apreciar las especificidades que exige la misma y examina los aspectos epistemológicos del tema y los referidos a la metodología de investigación científica de dicha disciplina. Se visualiza la escritura como proceso y como producto con la intención de identificar la complejidad del fenómeno en sus múltiples dimensiones. Finalmente, se resaltan las ventajas socioculturales y políticas que trae consigo el estudio de la argumentación escrita en la escuela y algunas formas a través de las cuales se puede potenciar.

En el Capítulo III, Didáctica de la argumentación escrita, se destaca el valor que representa la argumentación escrita en la vida académica, pero también en las 
actividades cotidianas del estudiante. Se describen proyectos que facilitan el desarrollo de la didáctica de la argumentación escrita, tales como: el Programa de Inteligencia Práctica (Sternberg, 1993; Gardner, 1995), el Programa de Filosofía para niños (Lipman, 1985) y se proponen distintos géneros argumentativos que facilitan esta tarea. Se remite a la filosofía para niños buscando superar el abismo abierto entre las prácticas discursivas y el conocimiento. Con fundamento en investigaciones precedentes a este trabajo, se propone el concepto de secuencia didáctica como una posibilidad para la producción de textos argumentativos en la escuela. Se presentan aspectos didácticos que favorecen el aprendizaje de la argumentación escrita y estrategias metodológicas cimentadas en algunos de los géneros argumentativos que contribuyen a la enseñanza y al aprendizaje de la misma. De igual manera, se plantean formas que ayudan al diseño, seguimiento y evaluación de secuencias didácticas para el desarrollo del tema en cuestión.

Como se sabe, la didáctica de la lengua y, en particular, de la argumentación escrita, es una preocupación constante en las escuelas francesa y ginebrina. Se pueden señalar los trabajos desarrollados por Bronckart (1970, 1985), Bronckart, Brun y Roulet (1991); de igual manera, los trabajos desarrollados por Dolz (1993, 1995), Dolz y Pasquier (1996). En el contexto español, se pueden destacar los trabajos propuestos por Camps (1995, 1997), Camps y Dolz (1995) y Álvarez (2001). La relación de los autores señalados con el libro reseñado es conceptualmente directa, la cual, se puede precisar de la siguiente manera:

- Bronckart (1970), aporta, por una parte, los estudios sobre análisis de los discursos como acción semiótica con sentido y como fundamento del desarrollo cognitivo; por otra, Bronckart (1985) brinda la teoría concerniente a las operaciones discursivas que realizan los interlocutores como operaciones psicológicas construidas en el desarrollo socio-cognitivo, cuya función es de orden comunicativo y pragmático. Y, con Bronckart, Brun y Roulet (1991), se promueve la noción de secuencia didáctica, la que lleva inmerso un proyecto de lengua o una propuesta de producción global (oral o escrita) que tiene una intención comunicativa y una propuesta de aprendizaje con objetivos y procesos de evaluación específicos.

- Dolz (1993, 1995), Dolz y Pasquier (1996) reivindican un "Decálogo para enseñar a escribir", buscando satisfacer las expectativas del estudiante y del profesor. Además, promueven los procesos argumentativos en la escuela y consideran de fundamental importancia: a) poner a los alumnos en contacto con situaciones argumentativas distintas y reales para que interioricen las características del texto argumentativo; b) prepararlos para elaborar argumentos y contraargumentos; c) llevarlos, no únicamente a la defensa de una tesis, sino al análisis y posible negociación de un compromiso; y d) ejercitarlos en ciertas estrategias lingüísticas propias de la argumentación. Camps y Dolz (1995) asumen los principios de las ciencias del lenguaje y la comunicación y las 
ciencias de la educación que sustentan el enfoque comunicativo y funcionalista en la enseñanza y el aprendizaje de la lengua. Camps $(1995,1997)$ propone que la didáctica de la lengua debe interesarse, además de otros aspectos, en: las características de determinado tipo de texto, la intervención del profesor, la interacción con los compañeros, los materiales, los niveles de la competencia comunicativa de los estudiantes y en la importancia de contextualizar las estrategias de escritura a las circunstancias del estudiante.

- Álvarez (2001) propone que la didáctica de la lengua debe partir de los contextos de acción y de los contenidos; insiste en que los contenidos científicos son campos teóricos de referencia, para transformarlos en contenidos de enseñanza o escolares, mediante la reformulación, la reducción y la simplificación; señala que transponer didácticamente los contenidos es definir y analizar el paso de los saberes teóricos (científicos y técnicos) o disciplinas de referencia (ciencias del lenguaje y de la comunicación) a los saberes enseñados o escolares. La transposición didáctica designa las transformaciones que experimenta un determinado saber en el momento en que se expone didácticamente.

Por su parte, en el mundo anglosajón, se destacan las aportaciones de Bereiter y Scardamalia (1977), Hayes y Flower (1980), Hayes (1996). En dichas publicaciones se reivindican los procesos en cuestión como operaciones cognitivas y metacognitivas complejas que ponen en juicio posturas operativas o instrumentales en el desarrollo de la lengua, la escritura y la argumentación. En cada caso de los mencionados en párrafos anteriores, hay aportes significativos que facilitan la configuración de la teoría expuesta en el texto Didácticas de la lengua y de la argumentación escrita. Dichas aportaciones provocan y promueven nuevas miradas del tema y la contextualización del mismo.

Aunque en varios países latinoamericanos se cuenta con investigaciones en torno a la argumentación escrita, la mayoría de ellas concluye en que el nivel de argumentación de los estudiantes es deficiente (Monzon, 2011). Así, hay que reconocer que en el actual estado del arte, aún se necesita fortalecer los estudios en esta área. Esto hace que el libro objeto de esta reseña adquiera relevancia para los estudiosos que asuman la didáctica de la argumentación escrita como investigación y como práctica.

$\mathrm{El}$ interés de este libro radica en que facilita la apropiación de conceptos claves como la teoría modular de la mente, el Programa de Inteligencia Práctica en la Escuela, el Programa de Filosofía para niños, la transposición didáctica y la secuencia didáctica para el desarrollo de la didáctica de la lengua y especialmente para el desarrollo de la argumentación escrita en la escuela. La integración de los aspectos señalados no solo facilita una mirada sistémica del problema sino, fundamentalmente, el asumir la argumentación en la escuela de manera exhaustiva, para proponer algunas alternativas de solución al problema mundial de la deficiente preparación de los estudiantes en educación básica, media, y superior en lo que respecta al tema en mención. 
Resulta relevante destacar que el libro que reseñamos está escrito en forma didáctica y permanentemente recurre a ejemplos, lo que hace que sea entendible tanto por el experto como por el neófito; asimismo, el rastreo histórico de los conceptos los hace más manejables y contextualizados. Además, la diversidad de opciones para el cometido hacen del documento una guía susceptible de contextualizarse en diferentes regiones y culturas; por tanto, está orientado no solo al experto sino -por su sencillez, claridad y coherencia- también hacia el estudiante que debe enfrentarse a los conocimientos de la lingüística, de la didáctica y específicamente a la teoría y a la práctica de la argumentación escrita. Permite navegar por los conceptos, las tensiones y las perspectivas de las didácticas de la lengua y de la argumentación escrita y hallar los fundamentos para comprender el complejo ámbito en el cual se articulan la educación con la lectura y la escritura, y que se revierte en el conjunto social para cualificar los niveles de desarrollo alcanzados por los proyectos educativos que orientan la institución educativa en Latinoamérica. 


\section{REFERENCIAS BIBLIOGRÁFICAS}

Álvarez, T. (2001). Textos expositivos-explicativos y argumentativos. Barcelona: Octaedro.

Bereiter, M. \& Scardamalia, M. (1977). The Psychology of write composition. Hillsdale, N. J.: Lawrence Erlbaum.

Bronckart, J. P. (1970). Teorías del lenguaje. Barcelona: Herder.

Bronckart, J. P. (1985). Les sciences du langage, un défi pour l'enseignement? Paris: UNESCO et Delachaux et Niestlé.

Bronckart, J. P., Brun, J. \& Roulet, E. (1991). Quelles directions de recherche pour la didactique du FLM? Etudes de Linguistique Appliquée, 84, 111-120.

Camps, A. (1995). Aprender a escribir textos argumentativos: Características dialógicas de la argumentación. En A. Camps \& J. Dolz (Coords.), Comunicación, Lenguaje y Educación. Enseñar a argumentar (pp. 51-63). Madrid: Edisa.

Camps, A. (1997). La especificidad del área de la didáctica de la lengua. Una visión sobre la delimitación de los contenidos de la enseñanza de la lengua y la literatura. En A. Mendoza (Coord.), Conceptos clave en didáctica de la lengua y la literatura (pp. 33-47). Barcelona: SEDLL / CIE/ Horsori.

Camps, A. \& Dolz, J. (1995). Introducción. Enseñar a argumentar un desafío para la escuela actual. En A. Camps \& J. Dolz (Coords.), Comunicación, Lenguaje y Educación. Enseñar a argumentar (pp. 5-8). Madrid: Edisa.

Dolz, J. (1993). La interacción de las actividades orales y escritas y la enseñanza de la argumentación. Comunicación, Lenguaje y Educación, 23, 17-27.

Dolz, J. (1995). Escribir textos argumentativos para mejorar su comprensión. En A. Camps \& J. Dolz (Coord.), Comunicación, Lenguaje y Educación. Enseñar a argumentar (pp. 65-77). Madrid: Edisa.

Dolz, J. \& Pasquier, A. (1996). Argumentarpara convencer. Una secuencia didáctica de iniciación al texto argumentativo para el primer ciclo de la Educación Secundaria Obligatoria. Gobierno de Navarra, Departamento de Educación y Cultura.

Gardner, H. (1995). Inteligencias múltiples. Barcelona: Paidós.

Hayes, J. (1996). A new framework for understanding cognition and affect in writing. En C. Levy \& S. Ransdell (Eds.), The science of writing: theories, methods, individual differences, and applications (pp. 1-27). Nueva Jersey: Lawrence Erlbaum Associates.

Hayes, J. \& Flower, L. (1980). Identifying the organization of writing process. En L.W. Gregg \& E. Steinberg (Eds.), Cognitive processes in writing (pp. 3-30). Hillsdale, N. J.: Lawrence Erlbaum.

Lipman, M. (1985). La utilidad de la filosofía en la educación de la juventud. Revista de filosofía y didáctica de filosofía, 3, 7-12.

Monzón, L. (2011). Argumentación: Objeto olvidado para la investigación en México. Revista electrónica de investigación educativa, 3, 2.

Ramírez, R. (2003). Inteligencia práctica en la escuela aplicada al desarrollo de la competencia argumentativa escrita. Hechos y Proyecciones del Lenguaje, 11, 29-51. 
Ramírez, R. (2004). La competencia argumentativa escrita en estudiantes colombianos de sexto grado de educación básica. Madrid: Universidad Complutense de Madrid.

Ramírez, R. (2005a). Aproximación al concepto de transposición didáctica, Folios. Segunda Época, 21, 33-45.

Ramírez, R. (2005b). La argumentación en la escuela. Hechos y Proyecciones del Lenguaje, $12,63-73$.

Ramírez, R. (2008). Breve historia y perspectivas de la argumentación. Pasto: Universidad de Nariño.

Sternberg, R. J. (1993). La inteligencia práctica en las escuelas. En J. Beltrán, V. Bermejo, M. D. Prieto \& D. Vence (Eds.), Intervención psicopedagógica (pp. 15167). Madrid: Pirámide. 